\title{
Percutaneous nephrolithotomy: Three-needle technique on two planes. Cury's technique
}

\author{
Carlos Abib Cury ${ }^{1}$, Analaura de Oliveira Cury ${ }^{2}$, Victoria Caroline Pagelkopf ${ }^{3}$, \\ Vinicius Ramos Bezerra de Morais ${ }^{3}$, Vitor de Almeida Fernandes ${ }^{4}$, Miguel Bonfitto ${ }^{4}$ \\ ${ }^{1}$ Professor, Urology Sector, São José do Rio Preto School of Medicine (Famerp), Brazil; \\ ${ }^{2}$ Medical Student, Votuporanga University Center (Unifev), Brazil \\ ${ }^{3}$ Medical Student, São José do Rio Preto School of Medicine (Famerp), Brazil; \\ ${ }^{4}$ Resident in Urology, São José do Rio Preto Base Hospital/Famerp, Brazil.
}

\begin{abstract}
Summary Introduction: Percutaneous nephrolithotomy is the main type of surgery indicated for kidney stones larger than $2 \mathrm{~cm}$. The present study describes a three-needle technique for percutaneous nephrolithotomy on two planes.

Surgical technique: The patient is first placed in the lithotomy position for cystoscopy, which guides the ureteral and urethral catheter. Next, the patient is placed in ventral decubitus for the three-needle technique. With the aid of the nephroscope, the first needle is positioned in the projection of the renal pelvis, the second needle is placed in the most posterior of the inferior calyces and the third needle is aligned with the other two at the most depressible point determined by the surgeon's index finger. After alignment in the topography of the third needle, an incision is made with the scalpel 1-2 cm perpendicular to the aponeurosis of the latissimus dorsi muscle. The second needle is replaced with a peridural needle, which is used to confirm the location of the inferior renal calyx and limit the depth of the Chiba needle inserted in the topography of the third needle, forming a $90^{\circ}$ angle with the peridural needle. The Chiba needle enables the passage of the guidewire and subsequent dilatation until the $30 \mathrm{~F}$ caliber for the passage of the Amplatz dilator, initiating the conventional procedure. Comments: The technique described has been used at our service for 15 years and has the advantages of less morbidity, fewer complications and less use of the nephrostomy tube. The technique is also easy to learn and highly reproducible.
\end{abstract}

KEY WORDS: Urology; Nephrolithiasis; Percutaneous nephrolithotomy.

Submitted 27 August 2019; Accepted 1 September 2019

\section{INTRODUCTION}

Lithiasis of the urinary tract is a common cause of urological morbidity, affecting approximately $12 \%$ of the population and with recurrences in approximately $50 \%$ of cases (1-4). For kidney stones larger than $2 \mathrm{~cm}$ in diameter, percutaneous nephrolithotomy had been the main treatment method since the first successful surgery described by Fernström and Johansson in 1976 (2-4). Percutaneous nephrolithotomy replaced open surgery for the treatment of these calculi, as the reduction in morbidity and improvement in the experience of surgeons led to the increased indication for this procedure $(5,6)$. According to the guidelines of the European
Association of Urology and American Urological Association, the percutaneous approach is indicated for renal calculi larger than $2 \mathrm{~cm}$ in diameter or calculi located in the inferior pole larger than $1.0 \mathrm{~cm}$ in diameter $(2,3)$.

This paper describes a novel three-needle technique for percutaneous nephrolithotomy on two planes.

\section{Surgical technique}

The patient is first placed in the lithotomy position. Cystoscopy is performed, followed by insertion of the ureteral and urethral catheter. The patient is then placed in ventral decubitus with the lower limbs slightly flexed for the onset of the three-needle technique.

1. With the aid of the fluoroscope, the projection of the renal pelvis is determined through cutaneous demarcation with Kelly forceps (Figures 1,2) in order to not expose the surgeon's hand to radiation.

2. The first needle $(21 \mathrm{G})$ is positioned in the projection of the renal pelvis previously demarcated by the Kelly forceps (Figure 3).

3. Next, the inferior calyces are located and reached through the injection of contrast through the ureteral catheter (Figure 4). The most posterior calyx is preferable due to its proximity to the puncture site.

4. The second needle is inserted in the calyx located in Item 3, obeying the same location steps using the Kelly forceps (Figure 5).

5. The two needles positioned in the pelvis and inferior calyx will serve as reference for the alignment of the third needle (Figure 6).

6. The third needle is positioned in the most depressible point between the $12^{\text {th }}$ rib and superior iliac crest determined by the surgeon's index finger on the axis of the first two aligned needles. The point is near the lumbar triangle and posterior axillary line (Figure 7).

7. In the topography of the $3^{\text {rd }}$ needle, an incision is made $1-2 \mathrm{~cm}$ perpendicular to the axis of the needles in the skin until reaching the thoracolumbar aponeurosis of the latissimus dorsi, which is also incised (Figure 8).

8. Next, the second needle in the projection of the inferior calyx is replaced with a peridural needle until reaching the lumen of the calyx, determined by urinary flow, thereby establishing the distance from the 


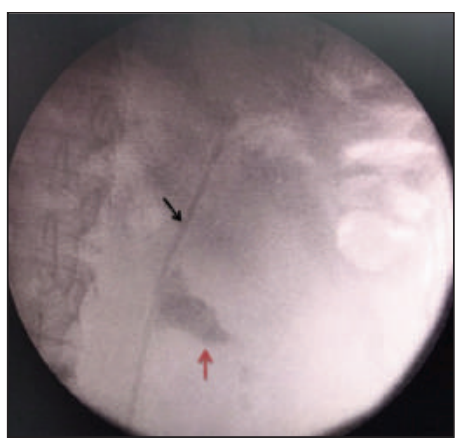

Figure 1.

$X$-ray showing location of calculus and catheter.

Figure 2.

(A) X-ray showing Kelly forceps in center of calculus with aid of fluoroscopy to demarcate projection of first needle to be inserted. (B) Cutaneous demarcation with Kelly forceps.

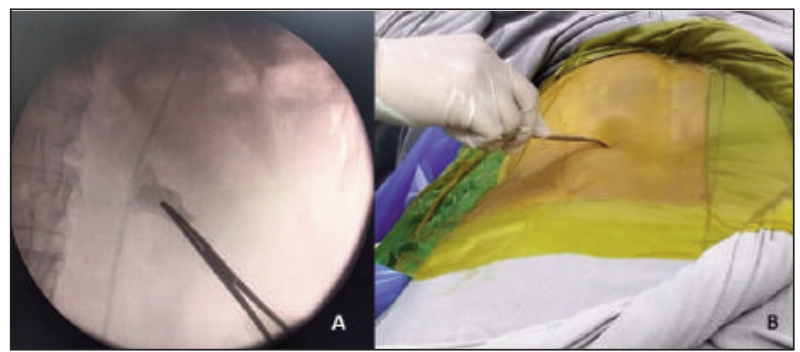

Figure 3.

(A) First needle positioned.

(B) Confirmation of site through fluoroscopy.
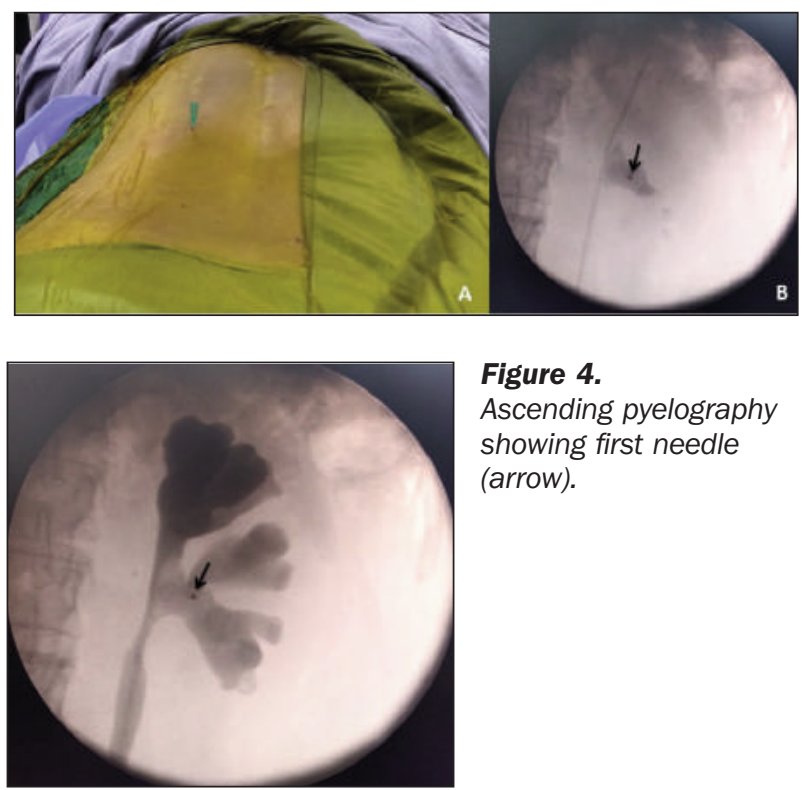

Figure 4.

Ascending pyelography showing first needle (arrow).

Figure 5.

(A) X-ray showing demarcation of puncture site for second needle in inferior calyces, opting for posterior calyx (B).

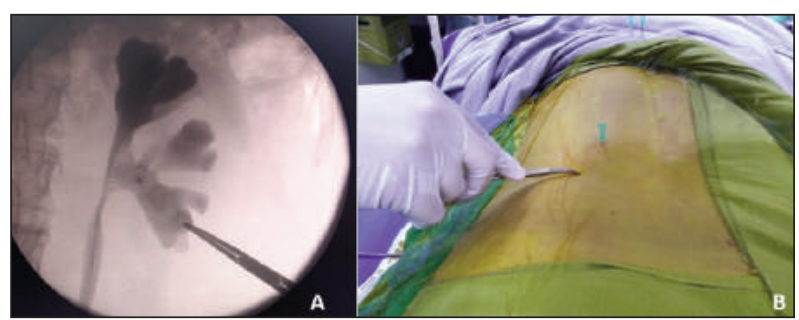

Figure 6.

(A) X-ray showing alignment guided by needles, establishing axis for puncture of third needle (B).

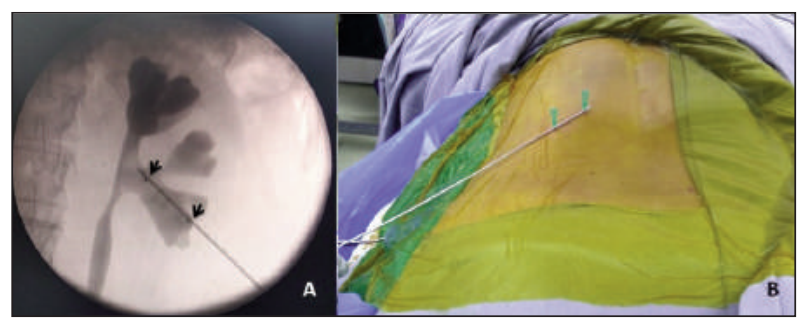

Figure 7.

(A) Most depressible point identified by index finger located between $12^{\text {th }}$ rib and upper iliac crest.

(B) Third needle inserted. (C) X-ray showing three needles and $(D)$ respective alignment.

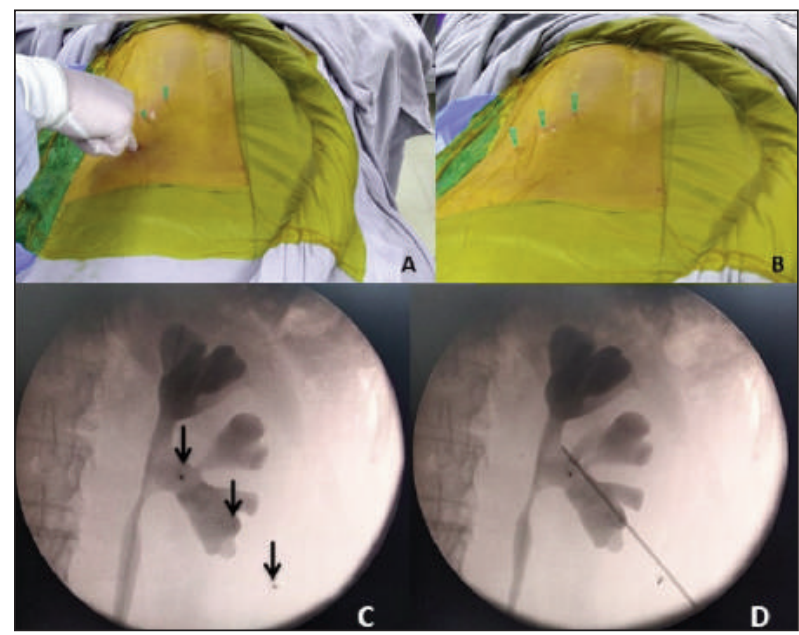

Figure 8.

Incision in

topography of third needle.

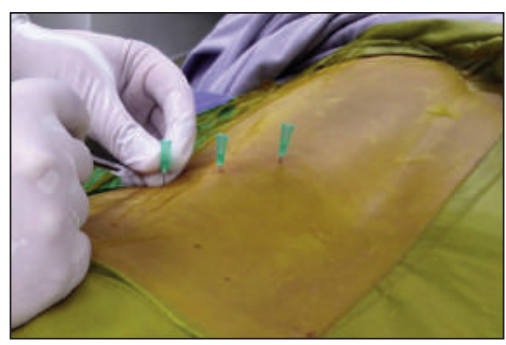

skin to the calyx, enabling the definition of the superficial and deep axes (Figure 9).

9. With the incision in the muscular and aponeurotic plane, the Kelly forceps are placed in the direction of the inferior pole of the kidney, opening the space in which the puncture and dilation of the percutaneous path will be performed (Figure 10).

10. In the opening achieved with the Kelly forceps, the surgeon's index finger verifies the path to toward the inferior pole of the kidney, which is easily moved (Figure 11).

11. The definitive puncture is initiated with the Chiba needle running the previously established path at an initial angle close to $45^{\circ}$ (individualized based on body mass and the orientation given by the surgeon's index finger). The Chiba needle should reach the depth determined by the peridural needle (Figure 12). 


\section{Figure 9.}

(A) Replacement of second needle (21 G) with peridural needle to reach calyx, determining puncture depth.

(B) Peridural needle positioned. (C) X-ray showing locations of first needle and peridural needle (arrows).

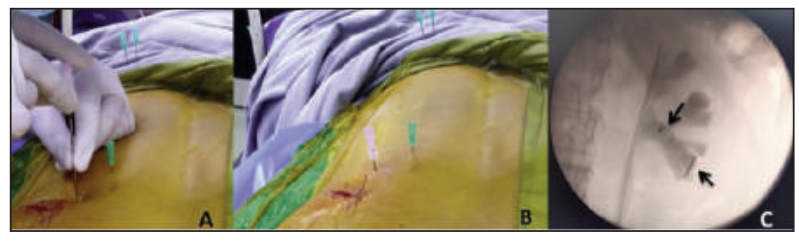

Figure 10.

(A) Insertion of Kelly forceps in direction of inferior renal pole, confirmed by X-ray (B).
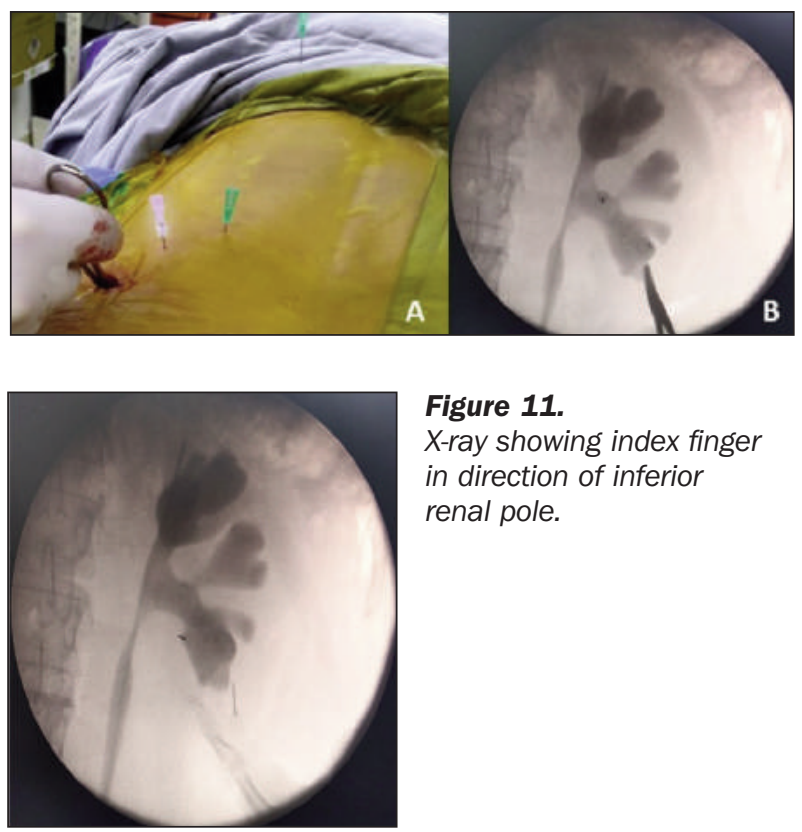

Figure 11.

X-ray showing index finger in direction of inferior renal pole.

Figure 12.

(A) Insertion of Chiba needle, traveling incision path. (B) X-ray showing Chiba needle in inferior extremity of peridural needle.

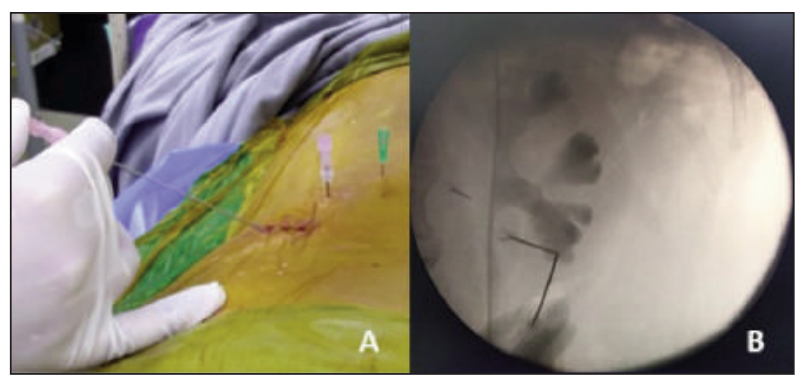

12. Upon reaching the extremity of the peridural needle, the Chiba needle is in the interior of the selected calyx, confirmed by the flow of urine (Figure 13).

13. Accessing the inferior calyx, the procedure is initiated by introducing the guidewire, followed by progressive dilation through a telescopic pathway with rigid instruments or individualized dilation to the 30F caliber, replaced by the Amplatz ${ }^{\circledR}$ dilator (Figure 14).

14. At this point, the entire procedure follows the con-
Figure 13.

Urine flow through lumen of Chiba needle.

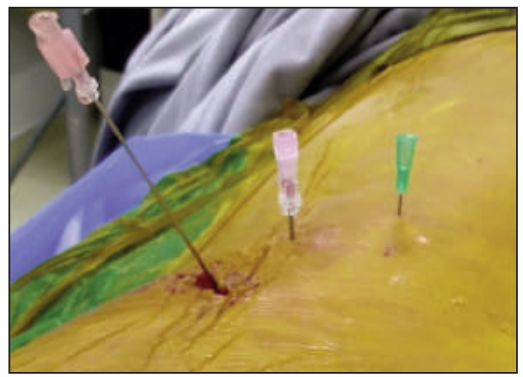

Figure 14.

(A) X-ray showing insertion of guidewire in calyx system.

(B) Dilation of path directed by guideline by telescopic route and initial and subsequent rigid instruments (C).

(D) Introduction of Alken's rod in dilator sleeve enveloping guidewire, which will be starting point for progressive dilation. (E) X-ray confirming location of Alken's rod.

(F) Introduction of last dilator with coupled Amplatz ${ }^{\circledR} 30 \mathrm{~F}$ dilator. (G) X-ray showing Amplatz ${ }^{\circledR}$ dilator positioned for continuity of procedure.

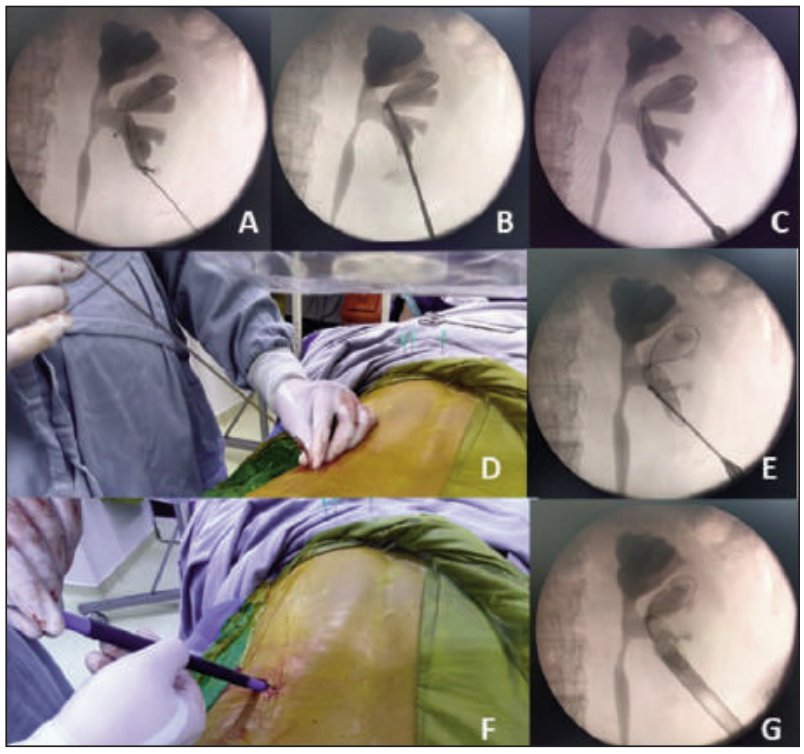

ventional standard for percutaneous nephrolithotomy, with the advantage that this slanted puncture enables better navigation of the nephroscope through the pelvis as well as the middle and upper calyces, thereby avoiding multiple punctures.

\section{Comments}

This technique have been used and improved over the course of 15 years at our service and has the advantage of a single access throughout the entire calyx system, thereby minimizing trauma to the parenchymatous tissue in comparison to a direct puncture, in which there is substantial parenchymatous injury when attempting to reach a neighboring calyx. In most cases, this technique avoids the use of a nephrostomy tube.

The three-needle technique is an alternative to conventional percutaneous nephrolithotomy. It is a novel treatment strategy for kidney stones that enables an approach on two planes in the prone position with the aid of a fluoroscope during the procedure. 
The technique obeys the Pythagorean theorem, which states that the sum of the squares of the catheti is equal to the square of the hypothenuse: $a^{2}+b^{2}=c^{2}$.

Cathetus "a" is the linear distance on the surface of the skin determined by the second needle to the third needle.

The hypothenuse " $\mathrm{c}$ " is defined by the surface of the third needle to the point of cathetus "b", determined by the second needle positioned in the inferior calyx. By obeying the theorem, the surgeon has greater control over the depth to which he/she wishes to insert the needle.

As kidneys are in an inclined position in relation to the spinal column at an angle of approximately $30^{\circ}$, the puncture in the inferior pole enables access to all calyces and the pelvis of the kidney, enabling the surgeon to navigate with the nephroscope through the renal excretory pathway, thereby avoiding further punctures. Access through the inferior pole diminishes the risk of injury to the renal vessels.

In summation, the technique described herein diminishes the use of the nephrostomy tube at the end of the procedure, leading to less postoperative pain and a shorter hospital stay.

\section{REFERENCES}

1. Teichman JM. Acute renal colic from ureteral calculus. New England Journal of Medicine. 2004; 350:684-93.

2. Guideline E.S. (2016). American Urological Association (AUA) Endourological Society Guideline SURGICAL MANAGEMENT OF STONES: AMERICAN UROLOGICAL ASSOCIATION/American Urological Association (AUA) Endourological Society Guideline Surgical Management of Stones, (April), 1-50.

3. Türk C, Skolarikos A, Neisius A, et al. Guidelines Associates: Donaldson JF, Drake T, Grivas N, Ruhayel Y. EAU guidelines on interventional treatment for urolithiasis. EAU Guidelines. Edn. presented at the EAU Annual Congress Barcelona 2019. ISBN 978-9492671-04-2.

4. Fernström I, Johansson B. Percutaneous pyelolithotomy: a new extraction technique. Scandinavian journal of urology and nephrology. 1976; 10:257-9.

5. Lee JY, Jeh SU, Kim MD, et al. Intraoperative and postoperative feasibility and safety of total tubeless, tubeless, small-bore tube, and standard percutaneous nephrolithotomy: a systematic review and network meta-analysis of 16 randomized controlled trials. BMC urology. 2017; 17:48.

6. Warmerdam GJ, De Laet K, Wijn RP, Wijn PF. Treatment options for active removal of renal stones. Journal of medical engineering engineering $\&$ technology. 2012; 36:147-55.

\author{
Correspondence \\ Carlos Abib Cury \\ Professor, Urology Sector, São José do Rio Preto School of Medicine (Famerp), \\ Brazil \\ Analaura de Oliveira Cury \\ Medical Student, Votuporanga University Center (Unifev), Brazil \\ Victoria Caroline Pagelkopf \\ Vinicius Ramos Bezerra de Morais \\ Medical Student, São José do Rio Preto School of Medicine (Famerp), Brazil \\ Vitor de Almeida Fernandes \\ Miguel Bonfitto \\ miguelbonfitto@gmail.com \\ Resident in Urology, São José do Rio Preto Base Hospital/Famerp, Brazil
}

